\title{
Practical Use of Emergency Tourniquets to Stop Bleeding in Major Limb Trauma
}

\author{
John F. Kragh, Jr., MD, Thomas J. Walters, PhD, David G. Baer, PhD, Charles J. Fox, MD, \\ Charles E. Wade, PhD, Jose Salinas, PhD, and COL John B. Holcomb, MC
}

Background: Previously we showed that tourniquets were lifesaving devices in the current war. Few studies, however, describe their actual morbidity in combat casualties. The purpose of this study was to measure tourniquet use and complications.

Methods: A prospective survey of casualties who required tourniquets was performed at a combat support hospital in Baghdad during 7 months in 2006. Patients were evaluated for tourniquet use, limb outcome, and morbidity. We identified potential morbidities from the literature and looked for them prospectively. The protocol was approved by the institutional review board.
Results: The 232 patients had 428 tourniquets applied on 309 injured limbs. The most effective tourniquets were the Emergency Medical Tourniquet $(\mathbf{9 2 \%})$ and the Combat Application Tourniquet (79\%). Four patients (1.7\%) sustained transient nerve palsy at the level of the tourniquet, whereas six had palsies at the wound level. No association was seen between tourniquet time and morbidity. There was no apparent association of total tourniquet time and morbidity (clots, myonecrosis, rigor, pain, palsies, renal failure, amputation, and fasciotomy). No amputations resulted solely from tourniquet use. However, six $(2.6 \%)$ casualties with eight preexisting traumatic amputation injuries then had completion surgical amputations and also had tourniquets on for $>2$ hours. The rate of limbs with fasciotomies with tourniquet time $\leq 2$ hours was $28 \%(75$ of 272$)$ and $>2$ hours was $36 \%(9$ of $25, p=0.4)$.

Conclusions: Morbidity risk was low, and there was a positive risk benefit ratio in light of the survival benefit. No limbs were lost because of tourniquet use, and tourniquet duration was not associated with increased morbidity. Education for early military tourniquet use should continue.

Key Word: Tourniquet, Hemorrhage, Resuscitation, Mangled extremities, Mass casualties.

J Trauma. 2008;64:S38-S50.
$\mathbf{H}$ emorrhage from injured limbs continues to be a leading source of battlefield death, ${ }^{1,2}$ and we recently showed in the current war that emergency tourniquet use improves survival rates in patients with major limb trauma. ${ }^{3}$ In response to the US Army design, testing, training, and fielding of battlefield tourniquets, ${ }^{4,5}$ all military personnel in theater carry tourniquets, and they are now common on the battlefields of Iraq and Afghanistan, both in the hands of medical and nonmedical personnel.

Although the US military is not alone in establishing procedures and equipment for the use of tourniquets in the prehospital environment by both medical and nonmedical personnel, ${ }^{6,7}$ tourniquet use remains controversial and not

Submitted for publication October 29, 2007.

Accepted for publication October 30, 2007.

Copyright (C) 2008 by Lippincott Williams \& Wilkins

From the US Army Institute of Surgical Research (J.F.K., T.J.W., D.G.B., C.E.W., J.S., J.B.H.), Fort Sam Houston, Texas; and Walter Reed AMC (C.J.F.), Washington, DC.

The opinions or assertions contained herein are the private views of the authors and are not to be construed as official or reflecting the views of the Department of Defense or United States Government. The authors are employees of the US Government. This work was prepared as part of their official duties and, as such, there is no copyright to be transferred.

Address for reprints: John F. Kragh, Jr., MD, USA Institute of Surgical Research, Bone and Soft Tissue Trauma Research Program, 3400 Rawley E. Chambers Avenue, Bldg. 3611, Room L82-16, Fort Sam Houston, TX 78234-6315; e-mail: john.kragh@amedd.army.mil.

DOI: 10.1097/TA.0b013e31816086b1 agreed upon by all authors, ${ }^{8-10}$ with some authors banning prehospital use of tourniquets altogether. ${ }^{11}$ Because we showed that tourniquets were lifesaving devices, the next important controversy regards tourniquet capacity to damage tissue and cause amputation. Because research in the human use of emergency tourniquets is limited, the morbidity controversy has been based more on speculation rather than actual data. Since 2003, we collected data regarding emergency tourniquet results, and this study is a continuation and amplification of those efforts (see Beekley in this supplement to Journal of Trauma).

We performed a prospective observational study at the United States combat support hospital in Baghdad, Iraq, of patients who had tourniquets applied in the field or in the emergency department (ED). Our objective was to measure tourniquet use and complications attributable to their use.

\section{METHODS \\ Study Design}

The protocol for this study was approved by the Brooke Army Medical Center institutional review board. The study period was from March 19 to October 4, 2006. This was a prospective observational survey with cohort and subgroup analyses. All patients at the combat support hospital who had a tourniquet of any type used in their emergent health care (prehospital, ED, or intensive care unit) were included in the study. Patients with tourniquets ready at the bedside, purposefully left loose, or whose first applied tourniquet was in the 
hospital operating room were excluded. Detainees and prisoners of war are restricted from research by military policies and were also excluded. No experimental interventions were made.

\section{Data Collection}

Data collected included patient's age in years, gender, limb involved (right or left limb, upper or lower extremity, limb regions [forearm, arm, leg, thigh]), patient nationality of origin, date of emergency tourniquet use, type of tourniquet used (name of commercial device or description of improvised tourniquet), duration of tourniquet use in hours, application time (time between injury and use) in minutes, setting of tourniquet application (prehospital or ED), indication of tourniquet use (life-threatening or non-life-threatening hemorrhage), mechanism of injury (such as explosive device, motor vehicle crash, or gunshot wound), injury type (such as blunt or penetrating trauma), treatment (including operative procedures, number of transfused units), Injury Severity Scores (ISS), Abbreviated Injury Score, systolic blood pressure, base deficit, International Normalized Ratio (INR), initial heart rate, injury description (e.g., traumatic amputation, open fracture, artery lesion, etc.), provider level of the person who applied the tourniquet (self, bystander, soldier, medic, nurse, physician), outcome (limb salvage, death), complications (necrotic muscle, compartment syndrome, nerve palsy), duration of follow-up, and problems related to application (device malfunction, malpositioning, exsanguination, or continued bleeding from distal limb, distal compartment syndrome, distal myonecrosis, nerve palsy under the tourniquet site). Because of the difficulty of distinguishing cause and effect in a group of patients predominated by the severely and multiply injured often with preexisting vascular injury, ${ }^{12}$ all mortality and possible tourniquet-related complications such as palsies were noted as all cause in that we did not associate cause and effect with tourniquets except for palsy and amputation or shortening. Palsies at the level of tourniquet application have been associated with high tourniquet pressure, and often are transient neuropraxias, whereas palsies at the level of the wound are traumatic injuries including such lesions as nerve transections. ${ }^{7,13}$ Prolonged emergency tourniquet use has been associated with surgical shortening of injured limbs, based on the surgeon's concern about prolonged ischemia. ${ }^{14}$ Consequently, many tourniquet morbidities are associated with tourniquet use, but frequently cannot be attributed to the traumatic injury itself versus the potential injury caused by the tourniquet. We had access to the electronic records of US military casualties in Iraq, Germany, and the United States.

\section{Definitions}

A tourniquet was defined as any limb constrictive device, whether improvised or commercially manufactured, used in an attempt to stop extremity bleeding.

We evaluated tourniquet use in two ways: we categorized patients on the basis of where their tourniquets were placed geographically (prehospital or in the ED) and when they were placed physiologically in relation to shock. ED tourniquet patients were those patients that had a tourniquet first placed on a limb in the ED, and the other patients were prehospital. We also included one intensive care unit patient in the ED group. We looked at tourniquets by numbers of devices used in safety analysis and by numbers of patients with tourniquets (irrespective of number of devices) for mortality.

Shock was defined as a weak or absent radial pulse in an uninjured limb without a tourniquet. Patients with tourniquets first placed after the onset of shock were analyzed as shock and all other patients were not shock, meaning that shock was not present before application of first tourniquet. This approach is consistent with the clinical definitions used by the Tactical Combat Casualty Care course, taught to all military medics. ${ }^{15}$

Purposefully loose tourniquet use was when it was placed around the limb but not tight in case the limb might bleed later. This type of use was a precaution including bedside tourniquets ready for use, but these patients were excluded from analysis.

Tourniquets were deemed medically indicated if the postoperative determination was that the injury justified tourniquet use. Tourniquets were deemed tactically indicated with needs such as care under fire, and unindicated use was when neither a medical nor a tactical reason could be determined for the tourniquet use. For example, open fractures with vascular injuries were categorized as vascular injuries. Inappropriate use included medically unindicated use or purposefully using the tourniquet as a venous tourniquet.

Tourniquets were defined as misplaced when the limb wound was too proximal for a tourniquet to fit between the wound and the groin or axilla without covering part of the wound, the tourniquet was atop the wound (without the wound being too proximal), or the tourniquet was distal to the most proximal wound.

We looked at effectiveness in two ways, (1) did the tourniquet stop the bleeding, and (2) if it stopped the distal pulse; in other words, was the tourniquet fully or partially effective. Fully effective tourniquet use required both that visible bleeding was controlled and that the distal pulse was palpably eliminated. Partially effective use was when the pulse persisted. Drainage of blood by gravity from a tourniquet limb was not in and of itself an indicator of ineffectiveness. If there was an amputation and no distal limb to have a pulse or if there was a vascular injury without a distal pulse, we used the "stop bleeding" criterion as being fully effective. Back-up was when one (or more) tourniquets were placed side by side to the first tourniquet after the first tourniquet was ineffective.

We looked for morbidities possibly associable with tourniquet use in the literature, ${ }^{13}$ and included amputation or stump shortening, ${ }^{14}$ palsy, ${ }^{16,17}$ myonecrosis, ${ }^{18}$ significant pain,,${ }^{19,20}$ clot, fasciotomy, ${ }^{21}$ acute renal failure, ${ }^{22}$ rigor, abscess,${ }^{23}$ blisters, abrasions, contusions, and pinching. ${ }^{13}$ 
An amputation was a traumatic injury (traumatic amputation), a procedure (debridement or completion amputation within 24 hours of injury), a possible morbidity if the tourniquet time was $>2$ hours (stump shortening revision amputation after 24 hours of injury), surgery for failed limb salvage ( $>24$ hours after injury), or surgery for improved prosthetic fitting. Significant shortening was when a joint (hip, knee, ankle, or elbow) was incorporated into the surgery. ${ }^{12}$

Intravascular clot included any clinically detected venous thromboses, emboli, or procedural thrombectomies associated with vascular repairs. Instances in which tourniquet application resulted in a regional nerve block were designated significant pain.

\section{Statistical Analysis}

Descriptive statistics were used for tourniquet use and resulting outcomes. For comparisons between subgroups with categorical data, significance was determined using $\chi^{2}$ or, when category counts were less than five, using Fisher's exact test. Subgroup analyses for continuous data were done with Student's $t$ test. All tests were two-tailed. Descriptive statistics were used to draw conclusions regarding the potential for improved doctrine, training, or devices to impact the care given to combat casualties. A $p \leq 0.05$ was the critical value for determining significance for all tests.

\section{RESULTS \\ Study Group Demographics}

Two hundred sixty-seven patients of 1,462 casualties admitted to the combat support hospital during the study period had prehospital or ED tourniquets. Excluded patients numbered 32. Fifteen detainees treated during the study period were excluded, and 22 patients were also excluded because of purposefully loose tourniquet use, and two patients met both detainee and purposefully loose criteria. The resulting study group was 232 patients $(8.2 \%)$ that had 428 tourniquets applied on 309 limbs.

The study group included 220 men and 12 women. The seven nationalities represented included 131 Iraqis, 95 Americans, 2 South Africans, and 1 each Kenyan, Nepalese, Briton, and Indians. The average patient age was 29 years (median, 28; range, 4-70). There were nine children $(<18$ years old) and one elderly person ( $>65$ years old). Follow-up averaged 18 days (range, 0.5-152 days; median, 7 days). Patients had one to four mechanisms of injury for each limb injury. The majority of injuries (210 of 336 or $63 \%$ ) were caused by explosive devises (232 explosions, 73 gunshot, 17 burn, 13 motor vehicle crash, 1 knife). The mean ISS was 15 (median, 10), and 41 patients had an ISS of 25 or higher indicating severe injury associated with high mortality.

The 309 limbs with tourniquets included 164 right limbs, 145 left limbs, 81 upper extremities, and 228 lower extremities. For the 428 total tourniquets, the number of limbs with one tourniquet was 203, 95 limbs had two tourniquets, 10 limbs had three, and one limb had five tourniquets (the fifth tourniquet was applied on arrival in Germany for rebleeding during transportation). Tourniquets in the study population were applied by lay persons including bystanders, soldiers, medics, nurses, physician assistants, physicians, surgeons, and only three times did the patient self-apply one. Although most tourniquets were placed by unknown persons, when known, most tourniquets were put on by the medics. The study period (199 days) averaged 1.2 tourniquet patients per day (232 of 199) and 2.2 tourniquets per day (428 of 199). Prehospital tourniquets, when known, were applied 0 to 75 minutes after injury with an average and median of 10 minutes.

The patients showed disturbed physiology associated with hemorrhage. The initial base deficit on presentation for 75 patients was -6 or less; a -6 value indicated substantial physiologic abnormality. Ten patients had initial heart rates of 0 , and 135 patients had tachycardia. Initial International Normalized Ratio on presentation was 1.5 or higher for 51 patients; a 1.5 value indicated coagulopathy. The number of transfused blood units of any type was 10 units or more for 104 patients; 10 constituted a massive transfusion.

\section{Indications for Emergency Tourniquet Use}

All but 12 tourniquets (in 12 patients with soft tissue injuries) of 428 (97\%) tourniquets were indicated, i.e., the wound required a tourniquet for medical or tactical reasons (tourniquet placement under gunfire for quick evacuation to prevent more casualties). Three of the 12 patients each had a limb that had a minor soft tissue wound in which a prehospital tourniquet was not medically necessary; however, the tactical reasons made tourniquet placement appropriate. For these three patients, the tourniquet times ranged 0.2 to 0.5 hours, and the tourniquets were fully effective. These three patients had no morbidities and were returned to duty promptly without surgery. In the other nine patients ( 9 tourniquets, $2 \%$ ), all patients required surgery to treat deep soft tissue injuries without named artery or vein transections. In these nine patients, tourniquets were converted to pressure dressings at ED presentation, no tactical reason was present at the scene to indicate tourniquet use, and there was no trial of a pressure dressing in the field. The indicated tourniquets were often with associated vascular injury; for the 309 limbs, there were 87 traumatic amputations, 86 vascular injuries, 81 open fractures, 45 soft tissue injuries, and one crush injury. One of the 428 tourniquets was used purposefully as a venous tourniquet in the ED for open type IIIB tibia and fibula fractures. This inappropriate use was corrected on the spot, and a fasciotomy was performed followed by a late belowknee amputation after a flap failure in the United States, but cause and effect determination cannot be made easily for the tourniquet and fasciotomy or amputation. This one patient had a medically indicated tourniquet and was the only one with inappropriate tourniquet use that had morbidity (Fig. 1). The death rate in those with inappropriate use was $0 \%$ ( 0 of 13 patients), compared with mortality of $14 \%$ (31 of 219) 


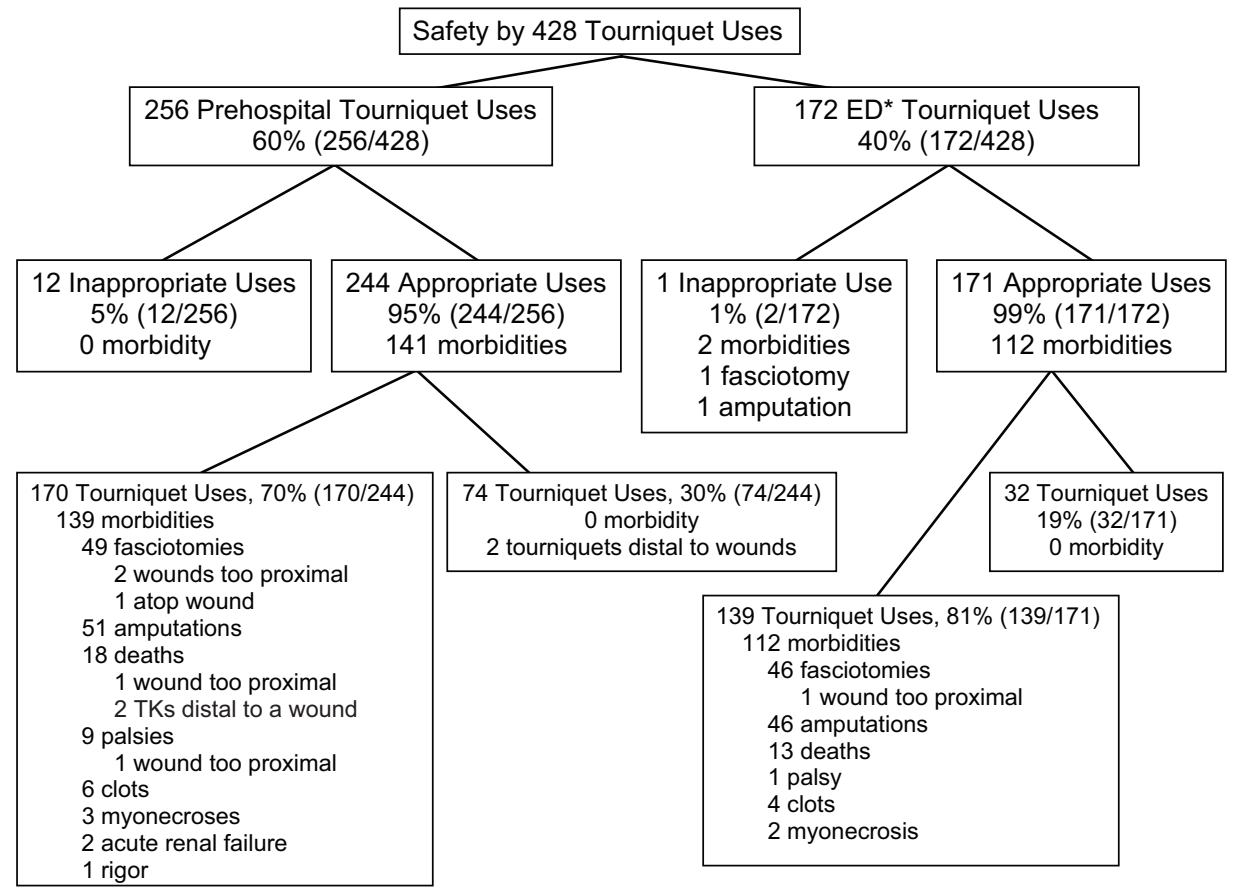

Fig. 1. Tourniquet safety by prehospital versus emergency department and inappropriate versus appropriate use. Use is by the number of tourniquet devices. Inappropriate use means not medically or tactically indicated or purposefully used as a venous tourniquet. Misplaced tourniquets are distal to or atop a wound, or the wound is too proximal for a tourniquet. The amputations in this figure are the sum of traumatic injuries, operations, and morbidities.

patients with appropriate tourniquet use. No impaled objects or neurologic injuries indicated tourniquet use.

\section{Some Morbidities Were Possibly Associable With Tourniquet Use}

Several types of morbidities have historically been associated to tourniquet use, and these potential complications were prospectively evaluated (Tables 1-6). The 10 clots included one deep venous thrombosis and nine thrombectomies, performed in conjunction with vascular surgery repair especially when the repair was distal. The vascular surgeon did not attribute these thrombectomies to tourniquet use. No pulmonary embolisms were detected in these patients, with computerized tomograms available to diagnose clinically suspected deep vein thrombosis and pulmonary embolism. Ten nerve palsies were diagnosed at the level of the wound in six and at the level of the tourniquet in four. The four palsies at the level of the tourniquet improved in the first hour to day after release, and only one had mild persistence at 6 days follow-up; all four were Iraqi patients. All four of these palsies at the level of the tourniquet were with prehospital use. Skin pinching, blisters, abrasions, or bruises were seen in seven limbs in five patients directly under the tourniquet area. Ninety-six fasciotomies were performed in the 232 patients as discussed below. Of the 87 amputation injuries, all had a surgical completion or debridements if the patient survived such (some did not live to get surgery) and 11 limbs had had failed limb salvage. In eight of the 98 limbs, there was also

\section{Table 1 Morbidities and Tourniquet Use}

\begin{tabular}{lc}
\hline \multicolumn{1}{c}{ Morbidity } & Number \\
\hline Amputation & 97 \\
Fasciotomy & 96 \\
Clot & 10 \\
Palsy & 10 \\
Myonecrosis & 5 \\
Acute renal failure & 2 \\
Significant pain & 1 \\
Rigor & 1 \\
\hline
\end{tabular}

Patients had 0-3 morbidities per limb. The amputations in the table are the sum of traumatic injuries, surgeries, and morbidities.

prolonged ( $>2$ hours) tourniquet use, a conventional threshold of prolonged use. ${ }^{13}$

\section{Tourniquet Time Was Associated With Amputation and Fasciotomy but Not Other Morbidity}

The tourniquet time was $<2$ hours, the limit of the so-called safe time, ${ }^{13}$ in $91 \%$ (269 of 297) of limbs with known tourniquet times (Table 2). The median tourniquet time was 1.0 hour, and the average was 1.3 hours. Tourniquet duration may have increased risk of only two morbidities, amputation and fasciotomy, and not other morbidities. There was no apparent association of total tourniquet time and morbidity with clots, myonecrosis, rigor, pain, palsies, and renal failure because, 0 of 10 clots, 1 of 5 myonecrosis morbidities, no rigor or pain case, 0 of 10 palsies, and 0 of 2 


\section{Table 2 Morbidity by Tourniquet Duration}

\begin{tabular}{lcrrrr}
\hline \multicolumn{1}{c}{ Total Tourniquet Duration } & 0 to $1 \mathrm{~h}$ & $>1-2 \mathrm{~h}$ & $>2-3 \mathrm{~h}$ & $>3-4 \mathrm{~h}$ & $>4 \mathrm{~h}$ \\
\hline Limbs with morbidity (\%) & 64 & 71 & 94 & 100 & 3 \\
Limbs with morbidity (N) & 98 & 84 & 16 & 5 \\
Limbs without morbidity (N) & 56 & 34 & 1 & 0 \\
\hline
\end{tabular}

* Tourniquet duration for 12 limbs was unknown. Patients had 0-3 morbidities per limb.

Table 3 Amputation Injuries in Patients With $>2 \mathrm{~h}$ Tourniquet Use

\begin{tabular}{clcccccc}
\hline Age $^{*}(\mathrm{yr})$ & Injury & Limb AIS & Surgery & Time $(\mathrm{h})$ & Lifesaving Intervention & Lived? & Follow-up (d) \\
\hline 18 & BEA & 3 & BEA & 4.7 & Yses & Yes & 6 \\
20 & BKA & 3 & BKA & 3.0 & Yes & Yes & 51 \\
19 & AKA & 4 & AKA & 2.3 & Yes & Yes & PH \\
19 & BKA & 3 & AKA & 14.0 & Yes & Yes & 42 \\
35 & R AKA & 4 & AKA & 8.0 & Yes & Yes & 9 \\
& L AKA & 4 & AKA & 8.0 & Yes & PH & PH \\
21 & R AKA & 4 & AKA & 2.1 & Yes & Yes & 1 \\
& L AKA & 4 & AKA & 2.3 & Yes & & PH \\
\hline
\end{tabular}

* There were 6 patients ( 8 traumatic amputations) with $>2 \mathrm{~h}$ tourniquet use.

BEA indicates below-elbow amputation; BKA, below-knee amputation; AKA, above-knee amputation; R, right; L, left; AIS, Abbreviated Injury Scale; $\mathrm{PH}$, prehospital tourniquet use; $\mathrm{H}$, hospital tourniquet use.

\section{Table 4 Transient Palsies in Patients With Tourniquet Use}

\begin{tabular}{|c|c|c|c|c|c|c|c|c|c|c|}
\hline $\mathrm{Age}^{*}(\mathrm{yr})$ & Nerve & Injury & Limb AIS & Time (h) & Lifesaving Intervention & Lived? & Follow-up (d) & Transient? & Level & Use \\
\hline 34 & Ulnar & Brach. a. & 3 & 1.95 & Yes & Yes & 3 & Yes & W & $\mathrm{H}$ \\
\hline 22 & Median & Brach. a. & 3 & 1.30 & Yes & Yes & 54 & Yes & W & $\mathrm{PH}$ \\
\hline 34 & Ulnar & Brach. a. & 3 & 1.01 & Yes & Yes & 10 & Yes & W & $\mathrm{PH}$ \\
\hline Unk & Ulnar & Ulna & 3 & Unk & Yes & Yes & 3 & No & W & $\mathrm{PH}$ \\
\hline 25 & Median & Elbow & 2 & 0.39 & Yes & Yes & 30 & Yes & W & $\mathrm{PH}$ \\
\hline 35 & All & Ulna & 3 & Unk & Yes & Yes & 7 & Yes & TK & $\mathrm{PH}$ \\
\hline 37 & All & Humerus & 3 & 0.88 & Yes & Yes & 6 & Yes & TK & $\mathrm{PH}$ \\
\hline
\end{tabular}

* There were 10 patients (10 limbs with palsies) with tourniquet use with 6 at the level of the wound (W) and 4 at the level of the tourniquet (TK).

Unk indicates unknown; all, all major peripheral nerves at the level; AIN, anterior interosseous nerve; CPN, common peroneal nerve; brach., brachial artery; v., vein; bones and elbow, fractures; AIS, Abbreviated Injury Scale; PH, prehospital tourniquet use; H, hospital tourniquet use.

\section{Table 5 Tourniquet Device Counts, Effectiveness, and Morbidity}

\begin{tabular}{|c|c|c|c|c|c|c|c|}
\hline Tourniquet Name & Patients; $\mathrm{N}^{*}$ & Devices; $\mathrm{N}^{*}$ & Limbs; $\mathrm{N}^{*}$ & Effective; N (\%) & Ineffective (\%) & Morbidity; $\mathrm{N}^{\star}(\%)$ & Back-Up (\%) \\
\hline CAT & 156 & 210 & 202 & $166(79)$ & $44(21)$ & $43(21)$ & 5 \\
\hline EMT & 91 & 115 & 115 & 106 (92) & $9(8)$ & $9(8)$ & 0 \\
\hline SOFT & 50 & 62 & 61 & $41(66)$ & $21(34)$ & $20(33)$ & 2 \\
\hline $\mathrm{RMT}$ & 2 & 2 & 2 & $0(0)$ & $2(100)$ & $2(100)$ & 0 \\
\hline London bridge & 1 & 1 & 1 & $1(100)$ & $0(0)$ & $1(100)$ & 0 \\
\hline Improvised & 15 & 16 & 15 & $4(25)$ & $12(75)$ & $12(80)$ & 17 \\
\hline
\end{tabular}

16 tourniquets were improvised from cravats, dressings, a string, a belt, a cord, a band, and an intravenous tube; 14 tourniquet types were unknown.

* Patients had 1-4 devices of 1-4 types on 1-4 limbs with 0-3 morbidities. A tourniquet that required another or more tourniquets on the same limb was said to require back up. There were 35 limbs where the effectiveness was unknown.

CAT indicates Combat Application Tourniquets; EMT, Emergency Military Tourniquets; SOFT, Special Operations Forces Tactical Tourniquet; SATS, Self-Applied Tourniquet System; RMT, Ratcheting Medical Tourniquets. 


\section{Table 6 Effectiveness of Tourniquets by Limb Region}

\begin{tabular}{|c|c|c|c|c|c|}
\hline Body Region & Patients; N & Limb Regions; N & Tourniquets; N & Effective; N (\%) & Ineffective; N (\%) \\
\hline Forearm & 9 & 9 & 13 & $12(92)$ & $1(8)$ \\
\hline Arm & 62 & 71 & 97 & 79 (81) & 18 (19) \\
\hline Leg & 22 & 27 & 32 & $32(100)$ & $0(0)$ \\
\hline
\end{tabular}

* Patients had 1-4 limbs injured with 1-4 tourniquets used per limb; 3 patients had tourniquets on their ipsilateral thigh and leg. There were 8 limbs with unknown tourniquet effectiveness.

renal failure cases, occurred in the 28 limbs with a total tourniquet time of more than 2 hours. The proportion of limbs with a tourniquet time of $>3$ hours that had an amputation injury surgically shortened was $62 \%$ (5 of 8 ), which is a lower proportion and lesser surgery than the amputation rate of "about 80\%" cited by Chisholm from the Civil War, the only comparable data set. ${ }^{24}$ The shortening rate of limbs with $\leq 2$ hours tourniquet duration was $87 \%$ (67 of 77 limbs, 14 limbs were not shortened because the patient died before surgery was possible), and of these, 58 limbs were shortened because of the injury, six because of failed vascular salvage, and three for better prosthetic fitting. The significant shortening rate (including a hip, knee, ankle, or elbow joint) was 19\% (15 of 77 limbs) for reasons unrelated to the tourniquet. The rate of limbs with fasciotomies with tourniquet time $\leq 2$ hours was $28 \%$ ( 75 of 272 ) and $>2$ hours was $36 \%$ (9 of 25 ). All nine fasciotomies after 2 hours of tourniquet duration were done prophylactically without evidence of compartment syndrome. One limb after 2 hours of tourniquet use did not have a fasciotomy, but did not develop compartment syndrome. Significant pain was seen in only one of 232 patients, and this patient had a traumatic above elbow amputation and tourniquet use of 1.35 hours with a ratcheted device and required an urgent regional block. Mortality was not associated with tourniquet duration; all 31 deaths occurred within 4 hours or less of tourniquet use (54 hours total tourniquet time for 31 patients and 47 limbs). There were 12 limbs (10 patients) with unknown tourniquet times, with no difference in outcomes in these 10 patients ( 8 of 10 patients survived, and 5 of 12 limbs were amputated). Of the 97 casualties with amputations, six had tourniquets for $>2$ hours in eight limbs that were lifesaving in all six patients. Only one limb was shortened to include a joint, from below-knee amputation to an above-knee amputation (Table 3, Fig. 2).

\section{Tourniquet Misplacement on Limbs Was Associated With Morbidity and Mortality}

We looked at placement of tourniquets in four ways: where the tourniquet was placed appropriately proximal to the wound, where it was placed distal to the most proximal wound that was not seen by the applier, where it was placed atop the wound but there was enough proximal room for it, and where the wound was too proximal for normal tourniquet placement proximal to the wound. Appropriately positioned tourniquets proximal to the limb wounds were seen in 417 of
428 tourniquet uses (97\%). Tourniquets distal to the most proximal limb wound numbered five on four limbs in four patients; two of these four patients died. A tourniquet was placed atop the wound in another patient; this patient had the

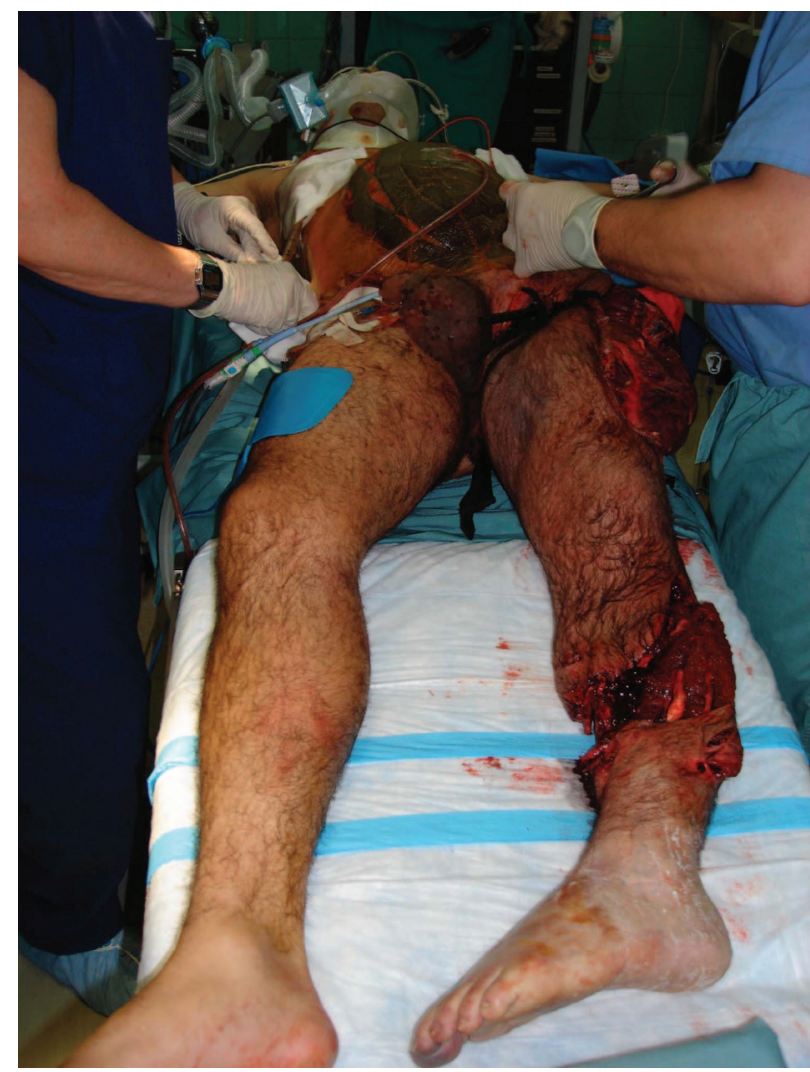

Fig. 2. Photograph of patient with multiple injuries and a tourniquet used on the thigh. The patient had a proximal femur fracture with femoral artery and vein transection and a near-complete below-knee amputation. The only left leg structures partially intact were in the deep posterior compartment. Other potentially lethal injuries required massive transfusion, emergency department thoracotomy and aortic cross clamping, open manual cardiac massage, three cardiac electroconversions, emergency laparotomy and ligature of the common iliac, femoral arteries and veins as hemorrhage control measures, and an intensive care unit respite to correct coagulopathy before the tourniquet could be attended and amputation done for the ischemic limb sequelae. This patient had a transfemoral amputation performed after 14 hours of tourniquet application. The patient survived. 
pulse persist and then had a fasciotomy, and lived. The wound was too proximal for the tourniquets to fit properly in four patients (four limbs, five tourniquets), and the tourniquets slowed but did not stop bleeding; one patient died, two had fasciotomies, and one had a nerve palsy. Despite the tourniquets being used for injuries they were not designed to treat, the $75 \%$ survival of patients with injuries that were too proximal for normal tourniquet use shows that tourniquets were useful when no better alternative treatment was available, as the most proximal of vascular injuries are the most lethal of limb injuries. These four proximal wounds were in the transition zone between compressible to incompressible lesions. Additionally, the type of tourniquet was not associated with improper application. Overall in casualties with misplaced use, mortality was 4 of 9 or $44 \%$.

\section{The Association of Tourniquet Ineffectiveness and Morbidity and Mortality Was Unclear}

Considering the effectiveness by stopping the bleeding or the distal pulse, morbidity, and mortality showed a pattern. The 44 patients with continued bleeding had morbidity or mortality $61 \%$ ( 27 of 44$)$ of the time ( 21 fasciotomies, 15 amputations, 4 deaths), four palsies, two myonecroses, one clot), and the 43 patients with persistent pulses had morbidity $51 \%$ (22 of 43) of the time (19 fasciotomies, 3 amputations, 1 death, 1 clot, 1 palsy). Total ineffectiveness (continued bleeding) was worse than partial ineffectiveness (persistent distal pulse) in that $61 \%$ and $51 \%$ had morbidity and $9 \%$ and $2 \%$ mortality rates, respectively. Ineffective tourniquets were associated with this clinical progression: persistent pulse, venous congestion, venous distention, rebleeding after a period of hemorrhage control, expanding hematomas, compartment syndrome, fasciotomy, and death, but this progression led only to 4 deaths. The persistent distal pulse risked high morbidity and low mortality, whereas persistent bleeding risked both high morbidity and high mortality.

All seven expanding hematomas were seen when the pulse persisted, but no expanding hematomas were seen when the tourniquet effectively stopped the distal pulse. Expanding hematomas are important because they are associated with continued blood loss, compartment syndrome, infections, and additional surgery such as fasciotomy and debridement. No gas or dry gangrene was seen. No cases of tourniquet necrosis or abscesses occurred. One limb with a traumatic amputation had an under tourniquet crush injury to the skin caused by the shearing of a bungee cord improvised tourniquet, but this skin injury was in the zone of injury and was amputated with the limb at a forward surgical team.

\section{Effectiveness: Combat Application Tourniquet Was the Best Prehospital Tourniquet and EMT Was the Best ED Tourniquet}

In general, the reasons why tourniquets were ineffective (continued wound bleeding or persistent distal pulse) included device narrowness relative to the limb girth, incorrect usage, and device breakage. The most commonly used tourniquet was the Combat Application Tourniquet (CAT) at 49\% of 428 uses (Table 5). For the tourniquets used for more than one patient, the CAT was the second most effective tourniquet at 79\%. The most effective was the Emergency Medical Tourniquet (EMT, 92\%). Decreasing effectiveness rates corresponded with tourniquet width as the EMT is widest, the CAT second widest, the Special Operations Forces Tactical Tourniquet (SOFTT) third, and the others as a whole were least wide. Although the EMT tourniquet was the most effective, it is not designed for prehospital medic use and is only available at higher levels of care. Improvised tourniquets were ineffective $67 \%$ of the time (10 of 15 limbs, 15 patients, 16 tourniquets) with 10 morbidities ( 6 amputation injuries, 3 fasciotomies, 1 palsy), and seven limbs continued to bleed. The wider improvised tourniquets (cravats and windlass type, especially when two were used side by side) were effective in $42 \%$ (3 of 7) of limbs, whereas the narrower ones (strings, i.v. tubing) were effective in 25\% ( 2 of 8 ) of limbs. The mortality rate in patients with improvised tourniquets was $0 \%$ (0 of 15), and the amputation rate was $40 \%$ ( 6 of 15, all 6 were traumatic amputations). Tourniquet ineffectiveness, (visible bleeding or distal pulse remained), and morbidities corresponded. Eightythree of $309(29 \%)$ limbs were still bleeding upon presentation or had a persistent distal pulse, and 10 patients were pulseless and receiving chest compressions on arrival and stopped bleeding prehospital after they had exsanguinated.

\section{Practical Issues Related to Tourniquet Effectiveness and Ineffectiveness}

Of the 309 limbs with tourniquets, $82 \%$ (167 of 203) with only one tourniquet used were completely effective, and 92\% (97 of 106) limbs with two or more tourniquets (sideby-side) were completely effective. Tourniquets were used side by side and only when one did not stop the bleeding.

Of the 83 limbs still bleeding on ED presentation, 43 limbs had an ineffective tourniquet (bleeding persisted or pulse persisted) uncorrected before arrival at the hospital, but most of these tourniquets in the 43 limbs had stopped the bleeding yet had a persistent distal pulse. There were many cases of minor amounts of dark blood oozing briefly from distal residual limbs after effective tourniquets were placed indicating simple drainage of blood from the limb with gravity, and these assessments were noted by senior clinicians as not being rebleeding or failures of tourniquets although this did cause some concern among other clinical personnel.

Effectiveness rates varied whether the tourniquets were used prehospital, in the ED, or both prehospital then ED. The prehospital effectiveness rate was 76\% (152 of 199 tourniquets for 51 limbs). The ED effectiveness rates was $86 \%$ (54 of 63 tourniquets for $171 \mathrm{limbs}$ ). The effectiveness rate for those tourniquets used both prehospital and ED was $76 \%$ (126 of 165 tourniquets for 79 limbs); eight limbs had unknown effectiveness of tourniquets. Tourniquet effectiveness varied by the limb region where the tourniquet was applied 
and was lowest in the thigh and highest for the leg (Table 6). Four patients had thigh and arm injuries (4 limbs with 5 tourniquets) where the wound was too proximal for normal tourniquet use, but four of these tourniquets stopped the bleeding yet the distal pulse persisted. There were seven patients (9 limbs, 15 tourniquets used) that had their tourniquet placed over Hunter's canal in the distal thigh, and the effectiveness of these tourniquets was 67\% (10 of 15). The distal Hunter's canal is deep to the adductor tendons of the thigh and protected from compression by the medial condyle of the femur, so that the superficial femoral artery at this point is difficult to compress. Three limbs ( 3 patients) had tourniquets on the leg and thigh. The effectiveness rates were concordant with our laboratory testing, which showed device width (relative to limb girth) was important, and there were no unexpected effectiveness results. ${ }^{4}$

Of the 309 limbs, the first tourniquet was effective (partially or completely) in 53\% of the time (164 of 309), whereas side-by-side use of a second or more tourniquets next to the first was effective an additional 34\% (106 of 309) for an overall effectiveness rate of $87 \%$ (270 of 309).

Ineffectiveness was caused by different issues: inadequate tourniquet design (too narrow), incorrect use (upside down), misplaced use (too distal to the wound, the wound was too proximal for tourniquet use) or a broken device. The device width (relative to limb girth) was the commonest reason for tourniquet ineffectiveness. There was one tourniquet placed incorrectly (one EMT placed upside down making the running end inflate instead of the cuff). There was one patient (one limb) that had a provider purposefully use a venous tourniquet in attempt to decrease risk of a palsy (the EMT tourniquet was soon inflated more to rid the distal pulse). Six tourniquets broke (three EMTs leaked, two EMT tube twist caps fell off, one CAT bar broke). Furthermore, two pneumatic EMTs required rotation of the clamp to become perpendicular to the bladder so that it could open but these two events did not result in ineffective uses just difficulties removing the tourniquet. One CAT bar was slippery when covered in blood, the device was ineffective, had to be backed up, and the patient died prehospital. Self application led to ineffective use in one of three times and difficulty pulling hard enough to properly tighten the tourniquet was reported by the patient; he could not tighten a thigh CAT, but his medic put a CAT on his leg effectively then removed the thigh CAT. The two effective self-applications were to a leg and a forearm in two separate patients.

\section{Under Tourniquet Padding and Transportation Were Associated With Looseness and Rebleeding}

Padding (such as the patient's clothes) under the tourniquet was associated with tourniquet looseness, but padding also lessened risk of blisters and pinching. Tourniquets became looser with limb or patient manipulation such as clothing removal; limb elevation, by hip flexion (translation of quadriceps and hamstrings in opposite directions with skin shearing under tourniquet); decreased limb girth caused by incremental exsanguination; and patient transfers to and from gurneys and transportation to and from vehicles. Retightening by providers was frequent after patient and tourniquet rechecks. Twelve of the 13 loose tourniquets were prehospital tourniquets. Two of the 13 patients with loose tourniquets died. The one hospital tourniquet that was loose became loose while a patient was transferred from a gurney, and it was retightened on the spot. One patient had a prehospital tourniquet removed that in hindsight was premature. This patient had an isolated injury to his foot, sustaining partially amputated toes and scything injuries to the four digital arteries. This patient had a tourniquet placed for difficult to control bleeding in the field, but the tourniquet was removed at a prehospital aid station by a medical officer. This patient reported bleeding throughout his evacuation to the hospital with pools of blood in the field, at the aid station, on the transport vehicle, and on the ED floor as he progressively hemorrhaged. On arrival in the ED, he was in shock (BP 100/60 mm Hg, pulse 143 on presentation) and required significant resuscitation (6 units of blood products transfused). Elevation and a pressure dressing in the ED helped slow but not stop the bleeding. In surgery, effective hemostasis required individual digital artery ligation. This case is similar to several cases in the civilian extremity hemorrhage study by Dorlac et al. ${ }^{25}$ Discontinuance of a prehospital applied tourniquet occurred only once in 282 prehospital tourniquet uses (257 limbs, 194 patients).

\section{Tourniquet On and Off Times Need Improved Documentation in Compliance With Doctrine}

Compliance of recording tourniquet application times was poor for both on and off times. Of the 309 limbs, 14\% (43 limbs) had the tourniquet application time, that is, on time, recorded in accordance with standard Army training or recorded on the field medical card. Based on operational data concerning each incident and gathered information from the medics who transported the patient, we estimated the application time for patients when data were available. The tourniquet removal time, that is off time, was recorded directly by the authors or recorded from interviewing other providers. No anesthetic record had the tourniquet off time recorded despite this being a routine practice during elective, peacetime operations.

\section{DISCUSSION \\ Battlefield Emergency Tourniquet Use Risks Morbidity Infrequently in Our Experience}

The main findings of the present study are that low risk of morbidity, and given their demonstrated capacity to save lives, a positive risk benefit ratio exists for tourniquets in our battlefield experience. ${ }^{3}$ Historically, there are many anecdotes of devastating complications associated with emergency tourniquet use such as clots, palsies, and blisters., ${ }^{40,13,26,27}$ Tourniquetrelated complications are well documented in animal studies. ${ }^{28}$ However, there are only two retrospective studies on emergency 
tourniquet use. ${ }^{7,29}$ The injuries in which emergency tourniquets are used makes it difficult to differentiate between direct muscle injury and those directly related to tourniquet use. Surgeons caring for the patients in this study had an appropriately low threshold for performing prophylactic fasciotomies because of concern for tourniquet associated limb swelling ${ }^{19}$ during the long air evacuation flights of US patients to Germany. Six patients with eight completion amputations had $>2$ hours of tourniquet use. However, in these cases other dominant factors were evident. In some of these cases, the decision to prolong tourniquet use was necessary, so that other potentially lethal injuries could be treated.

Of the 97 casualties with amputations, six had tourniquets for $>2$ hours in eight limbs that were lifesaving in all six patients. Only one limb was shortened to include a joint, from below-knee amputation to an above-knee amputation. The reason for shortening was complex and included a 14 hours tourniquet time, but also the shortening was at the level of a superficial femoral artery transection and open femur fracture, the laparotomy entailed ligation of the common femoral artery and vein, splenectomy, diaphragm repair, and peritoneal packing for diffuse coagulopathic bleeding. Confounders adding to the ischemia and reperfusion of the extremity were profound hemorrhagic shock (base deficit of -19 on presentation to the ED), ED thoracotomy and aortic clamping, 2 episodes of open cardiac massage, massive resuscitation and transfusion, coagulopathy, and staged damage control operations. The 14 hours of intensive care between the operations helped complete the resuscitation and normalize the casualty's coagulopathy. The patient survived. We think that the tourniquet in this case was lifesaving (Table 3, Fig. 2) We cannot relate cause (traumatic amputation, ligation of vessels, tourniquet use, shock, coagulopathy) and effect of surgical amputation in this case, but shortening of the residual limb from a below-knee amputation to above-knee amputation was related to tourniquet use, ligation of vessels, and prolonged damage control resuscitation and surgery. In other cases, delayed transportation or transfer was a cause of prolonged tourniquet time (Table 3). In a classic article on vascular injuries in war wounded limbs, Debakey and Simeone had over an order of magnitude of cases more than the present study, and yet they could not use amputation as a clear outcome because of trouble with records and differentiating amputation as an injury, debridement, reconstructive surgery for prosthetic fitting, or failed limb salvage for vascular, musculoskeletal, or infectious reasons. "The survival or death of the limb, which at first glance might seem to be a critically objective test, actually is not: It does not permit a clear decision as to whether the therapeutic measures employed in a given case have influenced the results, and it can serve as a criterion only when sufficiently large numbers of cases are available for statistical evaluation". ${ }^{12}$ We have previously reported a case from Afghanistan that had more than 16 hours of tourniquet use and kept his upper extremity and returned to piloting helicopters. ${ }^{3}$
Tourniquets that are overtightened have been associated with palsies by damaging the peripheral nerves by compression, and these all occurred with prehospital use. The too loose tourniquets preponderantly occurred in the prehospital group. Beyond the too tight and too loose tourniquets, the inappropriate (including medically and tactically unnecessary) use also preponderantly occurred in the prehospital group, suggesting the difficulty in practicing medicine in this environment, and the continuing need for education and refinement of tourniquet training. However, complication rates were similar between the prehospital and hospital use, which indicates a positive risk benefit ratio. Improved compliance with current doctrine of recording tourniquet times on the patient or in the accompanying records would aid surgeons in managing patients.

\section{The Current Study Compares Favorably With Other Tourniquet Studies}

The current study is the largest study to date in terms of the number of patients and tourniquets used, and our data are concordant with prior experience. ${ }^{7,29}$ But, the present study advances knowledge by measuring substantial survival benefits and limited morbidity risks. Wolf and Adkins in 1945 reported three case vignettes and lessons learned from 200 cases in the Mediterranean theater from an US military hospital in World War II, but besides the vignettes, the 200 patients' data were summarized in one sentence. "The experience of our group indicates these complications [nerve paralyses and vascular thromboses] are very rare; in fact, an analysis of 200 random cases in which tourniquets had been applied shows that these complications did not occur in a single instance, unless they showed up after the patients left our hands, usually five to ten days after surgery". ${ }^{29}$ Lakstein et al. in 2003 reported a retrospective study from Israel of 91 patients (110 limbs, 110 tourniquets, 3 models of tourniquets), and had no deaths and few data comparisons. ${ }^{7}$ We saw tourniquet use rates 29 times that of Lakstein et al. (428 tourniquets/ 199days vs. 110 tourniquets/1461days); the average limb Abbreviated Injury Score of the present study (2.9) was higher than that of Lakstein et al. (2.6). Lakstein et al. were criticized by Husum et al. for being unable to show that tourniquets were useful, lifesaving, or worth the risk. ${ }^{11}$ Further, our study was bigger with more diverse patients as Lakstein et al. had 1 nationality, all men, no children, no elderly, and no civilians and had only prehospital tourniquets of fewer designs. Beekley reported a retrospective review from the same hospital as the current study. They had 67 patients and found four of seven patients who died without tourniquets were potentially salvageable (see Beekley in this supplement to Journal of Trauma). ${ }^{30}$ The most important factor in improving survival in our patients was attaining rapid hemorrhage control with an appropriately placed tourniquet, thus limiting risk of the negative 
effects of shock. Mabry et al. in 2000 called for wider use of prehospital tourniquets based on experience in 1993 from Somalia. ${ }^{31}$

\section{If a Tourniquet Is Ineffective, Additional Tourniquets Side-by-Side Can Improve Effectiveness}

Additional findings of this study are that tourniquets can be used side by side if one tourniquet is ineffective, and that side-by-side use effectively widens the compression and stops the distal pulse and is associated with fewer complications than single use with persistent pulse. Side-by-side use can improve effectiveness when a single wide tourniquet is unavailable. Improvised tourniquets as a whole were effective only $25 \%$ of the time and were less effective than commercially designed, wide tourniquets, but the improvised tourniquets as used in the current study were more effective than the two narrowest commercial tourniquets (SATS, M2).

Our results underscore the importance of vigilance in rechecking the patient and tourniquet as they can get loose during transport leading to incremental rebleeding, shock, and death. Loosening was increased by keeping clothing under tourniquets and by transportation. The goal of tourniquet use is to save lives, but the mechanical goal is to not only stop bleeding but to rid the distal pulse when present. By measuring how tourniquets work and do not work, our results yield a clearer understanding of how to better use tourniquets.

\section{The CAT Was the Best Prehospital Tourniquet and the EMT Was the Best ED Tourniquet}

The CAT was selected for issue to all deploying US warriors following studies performed at the US Army Institute of Surgical Research. ${ }^{4,5}$ In the current report, the overall effectiveness for the CAT, EMT and SOFTT was 79\%, 92\%, and $66 \%$, respectively. In our original report, the EMT was recommended for use in medical evacuation vehicles, forward surgical teams, and at combat support hospitals. Although the EMT effectiveness was highest of all devices at $92 \%$, it was the most expensive, the most fragile, and required the most training to use; for these reasons, it is not the best device for issue to every soldier or medic. The SOFTT was chosen as a substitute if the CAT was unavailable. The present study validates these choices with clinical data. In laboratory testing in human volunteers, the efficacy of the CAT, EMT, and SOFTT was each found to be $100 \% .^{5}$ As one would expect, our laboratory testing results were slightly higher than the real world results. The lower values in war are most likely attributable to the complexities of real world conditions and lack of experience with the application of the tourniquet versus the ideal conditions of the laboratory using well instructed volunteers. In laboratory testing, the effectiveness rate of the SATS was $44 \%$, whereas in the field it was $0 \%$ effective. Taken together, these facts reinforce the contention that any tourniquet to be used on the battlefield should first be shown to be $100 \%$ effective in the laboratory. ${ }^{4}$ Effectiveness is a function of device (its availability, user familiarity, and device width), and individual circumstances (limb girth and systolic blood pressure). ${ }^{5,32,33}$

\section{Successful Tourniquet Use Hinges on Devices Used, Training, Doctrine, and Transport Speed}

Tourniquet use success overall within a trauma system will be a function of devices used, tourniquet use training, evidenced-based doctrine, and rapid evacuation to surgical hospitals. These traits were present for our study, but tourniquets may not be advisable and may jeopardize limbs in settings of inadequate devices, little training, no doctrine, and slow evacuation. The change in military training doctrine contained in the Tactical Combat Casualty Care Course has driven the liberal use of tourniquets. ${ }^{15}$ When under fire, in darkness, during mass casualty situations, or to deal with multiple limb exsanguinations, more liberal use of tourniquets has been doctrinally espoused to prevent deaths by limb exsanguinations. Because prior teaching had labeled tourniquets as a method of so-called last resort, ${ }^{34}$ there was limited experience in which to understand when or if to use tourniquets. Studies on emergency tourniquet use previously came from war zones such as the US wars (World War II, Korea, Vietnam, and Somalia) or the experience of the Israeli forces, and had limited documentation. Tourniquets may be considered for paramilitary or civilian practice although isolated limb hemorrhage has a low mortality in civilian trauma patients. ${ }^{25,35,36}$ Tourniquets are now widely available on the battlefield, currently 1.5 million CATs have been sold to all customers, civilian and military, according to the manufacturer. The highest mass casualty rate for the study period was 28 patients presenting to the ED in a 25 minute period, and three of the 28 patients had tourniquets used and all 28 had limb injuries. Civilians are likely to see mass casualties where tourniquets may be of use. The current study quantifies the benefits and risks associated with tourniquet use so that individual practitioners and leaders of trauma systems can decide when or if to field tourniquets in support of their patients.

\section{Controversy: Exsanguination Risk, Tourniquet Science, Training, and Clinical Experience}

There persists in the literature the controversy whether emergency tourniquets should be banned or widely used. Historically, in the US Civil War, Spanish Civil War, and World War I, tourniquets were tried often and then lost favor because of ineffectiveness, but in World War II, Vietnam, Somalia, and Israel, tourniquets use has continued. ${ }^{7,23,27,29,31}$ The ineffective strap and buckle tourniquet that was standard issue in the US Military for five decades may have accounted for this clinical response, and with better tourniquet knowledge over the decades, a coherent move to better designed tourniquets on the battlefield occurred. The pertinent issues include use in patients at risk of lethal exsanguination, improved tourniquet science, training and doctrine, and clinical research and experience. If a patient population is not at risk of lethal limb exsanguination, then tourniquets cannot save 
lives as implied in the study of Lakstein et al., but our patients were at high risk and survival was improved. Tourniquet science, much of it published recently regarding elective orthopedic use, was limited in prior wars, and so how tourniquets work optimally is better understood now. Particularly, the interrelationship of tourniquet width, limb girth, and arterial occlusion is now well studied, yet clinical awareness is limited even in orthopedic surgeons. ${ }^{37}$ Given improved tourniquet science, more and better designed tourniquets are available today than previously. For more than a decade, the military refined training and doctrine based on laboratory testing, research projects, and clinical experience such as in Somalia. ${ }^{4,30,31,38}$ In contradistinction to the current military situation, patients of long past wars with longer evacuation times, treated by providers without tourniquet science using inadequate tourniquets without training and doctrine, may have faired worse than current patients. This explains why tourniquet problems were reported by experienced surgeons from the US Civil War, World War I, the Spanish Civil War, and early World War II. ${ }^{21-24}$ Articles and textbooks written by senior consultants at the end of tertiary referral patterns are common, influential, and long referenced, ${ }^{13,18,20,21}$ but they do not have the perspective of prehospital providers and prehospital data as noted in recent literature. ${ }^{7,30,31,39,40}$ A fresh look at emergency tourniquets is appropriate in a reassessment of the risks and benefits for selected patients and populations.

Limitations of the present study include its specific setting and population, which limit generalization. Although the care was given in a war torn Mideast country, the patients rapidly entered a quality healthcare system. Long delays in arrival to a surgical hospital, as seen in international aid hospitals like those supported by the International Committee for the Red Cross, would likely risk more amputations after tourniquet use in isolated extremity injured casualties than we measured. Because of the difficulty of distinguishing cause and effect in a heterogeneous group of severely and multiply injured patients often with preexisting vascular injury with severe extremity injuries, mortality, and morbidity were not associated with tourniquets except for four palsies and a single case of significant amputation shortening. This all cause association was conservative in that only a tourniquet abolitionist would associate traumatic toe and finger amputations with a tourniquet used on the arm or thigh, or would associate rigor in an uninjured limb with tourniquet use. Further, we used a conservative, mechanical definition of effectiveness where a loose tourniquet could be fully effective after the patient exsanguinated before presenting to us in that no bleeding or pulse persisted distally. We took a conservative approach to the research given the historical controversy. The halving of the mortality rate with prehospital use was associated with a $16 \%$ improvement in survival rate compared with ED use. This improvement yielded an estimated 31 lives saved.

Tourniquets are powerful tools for good and bad. Tourniquets are good in that they save lives when used at the right time, in the right way, but bad in that they can complicate care if used at the wrong time, and in the wrong way. Using tourniquets after extrication, transport, or shock, or distal to the wound caused increased mortality in our patients. The benefits far outweighed the risks in our experience with no limbs lost solely from tourniquet use. We saved an estimated 31 lives at the cost of one knee by using prehospital tourniquets compared with restricting use to ED. Tourniquets are a temporary measure allowing effective hemorrhage control and must be applied early, before the casualty is in shock to save lives. A tourniquet does not reverse shock, but it may attenuate shock and can gain the provider vital time to initiate effective resuscitation. We recommend continuing the policy of encouragement of emergency tourniquet use if the risk of injury is high, the probability of lethal limb hemorrhage is high, and there is systematic training of all at risk personnel. Policies of discouragement of tourniquet use may be appropriate in other settings, but in the current war will increase the death rate. Military and civilian leaders may use the reported experience herein to help decide how or when to use tourniquets by balancing the reported risks and benefits.

\section{Practical Recommendations Based on the Current Study's Findings}

- Tourniquet use before shock onset saves more lives than after shock; use them before extraction or transport.

- Use scientifically designed, laboratory tested, and clinically validated tourniquets.

- Use improvised windlass tourniquets when scientifically designed tourniquets are unavailable.

- Of tourniquets evaluated in this work, the CAT is the best prehospital tourniquet, and the EMT is the best ED tourniquet.

- Tourniquet education, training, and doctrine are vital and should be refined based on evidence.

- The goal of emergency tourniquet use is to stop bleeding and stop the distal pulse.

- Avoid tourniquet use over Hunter's canal near the knee as it risks ineffectiveness.

- Side-by-side use is useful to rid distal pulses and stop bleeding if one tourniquet is ineffective.

- Tourniquets work well proximal to the wound even on the forearm or leg and need not only be on the thigh or arm as sometimes recommended.

- Clothing about a tourniquet should be removed at the first opportunity to detect all wounds.

- Materials under a tourniquet should be removed at the first opportunity to avoid looseness.

Future research needs include matched cohort comparisons to confirm if tourniquets are lifesaving and to see if the findings apply to other populations. More clinical research is needed in first aid of patients with limb injuries. The only first aid device we know of that showed improved survival 
after limb injury was the Thomas splint, ${ }^{41,42}$ but now we now have convincing evidence that tourniquets can also improve survival. These data will inform the best possible care. Improved methods of early hemorrhage control are required to improve survival on the battlefield, and currently tourniquets are first aid tools that save lives.

\section{ACKNOWLEDGMENTS}

We thank Joanna G. Branstetter and Amy Newland for assistance in manuscript preparation, and John Jones for contingency testing.

\section{REFERENCES}

1. Bellamy RF. The causes of death in conventional land warfare: implications for combat casualty care research. Mil Med. 1984; 149:55-62.

2. Holcomb JB, McMullin NR, Pearse L, et al. Causes of death in U.S. Special Operations Forces in the global war on terrorism: 2001-2004. Ann Surg. 2007;245:986-991.

3. Kragh JF Jr, Baer DG, Walters TJ. Extended (16-hour) tourniquet application after combat wounds: a case report and review of the current literature. J Orthop Trauma. 2007;21:274-278.

4. Walters TJ, Mabry RL. Issues related to the use of tourniquets on the battlefield. Mil Med. 2005;170:770-775.

5. Walters TJ, Wenke JC, Kauvar DS, McManus JG, Holcomb JB, Baer DG. Effectiveness of self-applied tourniquets in human volunteers. Prehosp Emerg Care. 2005;9:416-422.

6. King RB, Filips D, Blitz S, Logsetty S. Evaluation of possible tourniquet systems for use in the Canadian Forces. J Trauma. 2006; 60:1061-1071.

7. Lakstein D, Blumenfeld A, Sokolov T, et al. Tourniquets for hemorrhage control on the battlefield: a 4-year accumulated experience. J Trauma. 2003;54(5 Suppl):S221-S225.

8. Navein J, Coupland R, Dunn R. The tourniquet controversy. J Trauma. 2003;54(5 Suppl):S219-S220.

9. Pillgram-Larsen J, Mellesmo S. [Not a tourniquet, but compressive dressing. Experience from 68 traumatic amputations after injuries from mines.] Tidsskr Nor Laegeforen. 1992;112:2188-2190.

10. Welling DR, Burris DG, Hutton JE, Minken SL, Rich NM. A balanced approach to tourniquet use: lessons learned and relearned. J Am Coll Surg. 2006;203:106-115.

11. Husum H, Gilbert M, Wisborg T, Pillgram-Larsen J. Prehospital tourniquets: there should be no controversy. J Trauma. 2004; $56: 214-215$

12. Debakey ME, Simeone FA. Battle injuries of the arteries in World War II: an analysis of 2,471 cases. Ann Surg. 1946;123:534-579.

13. Klenerman L. The Tourniquet Manual. London: Springer; 2003.

14. Coupland RM. War Wounds of Limbs, Surgical Management. Oxford: Butterworth-Heinemann; 1993:20-21.

15. National Association of Emergency Medical Technicians. Prehospital Trauma Life Support: Military Version. 6th ed. St Louis: Mosby; 2007.

16. Klenerman L. Tourniquet paralysis. J Bone Joint Surg Br. 1983; 65:374-375.

17. Middleton RW, Varian JP. Tourniquet paralysis. Aust N Z J Surg. 1974;44:124-128.

18. Watson-Jones R. Fractures and Joint Injuries. 4th ed. London: E. \& S. Livingston; 1955:121-122.

19. Konrad G, Markmiller M, Lenich A, Mayr E, Ruter A. Tourniquets may increase postoperative swelling and pain after internal fixation of ankle fractures. Clin Orthop Relat Res. 2005;433:189-194.

20. Klenerman L. The tourniquet in surgery. J Bone Joint Surg Br. 1962;44:937-943.

21. Böhler L. The Treatment of Fractures. New York: Grune \& Stratton; 1956:126-128.
22. Watson-Jones R. Traumatic uraemia. J Bone Joint Surg Br. 1948;30:233.

23. Jolly DW. Field Surgery in Total War. New York: Hoeber, 1941:24-25.

24. Chisholm JJ. A Manual of Military Surgery. Richmond, VA: West \& Johnson; 1861:140-143.

25. Dorlac WC, DeBakey ME, Holcomb JB, et al. Mortality from isolated civilian penetrating extremity injury. J Trauma. 2005;59:217-222.

26. Bellamy RF. Why is Marine combat mortality less than that of the Army? Mil Med. 2000;165:362-367.

27. Mabry RL. Tourniquet use on the battlefield. Mil Med. 2006;171:352-356.

28. Pedowitz RA. Tourniquet-induced neuromuscular injury. A recent review of rabbit and clinical experiments. Acta Orthop Scand Suppl. 1991;245:1-33.

29. Wolff LH, Adkins TF. Tourniquet problems in war injuries. Bulletin US Army Med Depart. 1945;37:77-84.

30. Sebesta J. Special lessons learned from Iraq. Surg Clin North Am. 2006;86:711-726.

31. Mabry RL, Holcomb JB, Baker AM, et al. United States Army Rangers in Somalia: an analysis of combat casualties on an urban battlefield. J Trauma. 2000;49:515-528; discussion 528-519.

32. Crenshaw AG, Hargens AR, Gershuni DH, Rydevik B. Wide tourniquet cuffs more effective at lower inflation pressures. Acta Orthop Scand. 1988;59:447-451.

33. Moore MR, Garfin SR, Hargens AR. Wide tourniquets eliminate blood flow at low inflation pressures. J Hand Surg [Am]. 1987; 12:1006-1011.

34. Emergency War Surgery. Washington, DC: Department of Defense, US Government Printing Office; 1975.

35. Office of The Surgeon General, US Army. Emergency War Surgery. 3rd US revision. Washington, DC: Department of Defense, USAMEDD Center and School, Borden Institute; 2004.

36. Langley DM, Criddle LM. The tourniquet debate. J Emerg Nurs. 2006;32:354-356.

37. Tejwani NC, Immerman I, Achan P, Egol KA, McLaurin T. Tourniquet cuff pressure: the gulf between science and practice. J Trauma. 2006;61:1415-1418.

38. Calkins D, Snow C, Costello M, Bentley TB. Evaluation of possible battlefield tourniquet systems for the far-forward setting. Mil Med. 2000;165:379-384.

39. Mallory M. Emergency treatment and resuscitation at the battalion level. Recent Adv Med Surg. 1954;1:61-67.

40. Tarpey MJ. Tactical combat casualty care in Operation Iraqi Freedom. Army Med Depart J. 2005;38-41.

41. Kirkup J. Foundation lecture. Fracture care of friend and foe during World War I. ANZ J Surg. 2003;73:453-459.

42. Gray HMW. The Early Treatment of War Wounds. London: Oxford University Press; 1919:49-63.

\section{DISCUSSION}

Dr. Paul E. Pepe (University of Texas Southwestern Medical Center, Dallas, TX): This ground-breaking study conducted by Colonel Kragh and his team regarding the potential complications of tourniquet use is highly commendable, not only in terms of the comprehensive results it provides us, but also in terms of the ambitiousness of the project. In a relatively short period of time, just over half a year, they collected dozens of prospectively defined data points on hundreds of patients, averaging more than two tourniquet applications per day. Assuming in this prospective study design that all consecutive tourniquet uses were captured, this is a remarkable fete just in terms of sheer work effort, let alone scientific contribution, particularly considering the setting, clinical environment, and the acute gravity of the medical conditions. 
If anything, there are so many data points discussed in the article that one almost loses the forest for the trees. In an attempt to be comprehensive, the authors reported more than a dozen pages of results. The authors carefully examined each nook and cranny of potential problems and examined them at every angle. At times, some of the results are descriptive in nature and many simple univariate associations are provided that are hypothesis-generating.

For example, they stated that prehospital application tended to be somewhat less effective than ED application. Although these findings may have reflected the care rendered by prehospital care providers, many of whom had very basic levels of training and most of whom could not be identified in retrospect, but it also may have reflected the tourniquet device employed. The EMT, not designed for prehospital care was used predominately in the ED, whereas the CAT was used more often in the prehospital setting. In other words, multiple factors may have affected the stated findings and one or more of these associative results may have been simple surrogate variables for the other findings. Nevertheless, the authors provide us with more data than we have ever had regarding this very timely subject. Those of us working in civilian trauma systems have been looking forward to this combat-based data initiative and the authors are to be thanked, not only for the data, but for the tremendous effort that they have put into reporting it.

One problem that does plague this study and the heretofore existing controversy of tourniquet application is the issue of time and duration of application. First of all, based on the previous work of others, the authors use a categorical scale of "greater than" or "less than" 2 hours to define "safe application" time. One could argue that one critical piece of data that this study could have offered us would be to examine morbidity versus time as a continuous variable.

Also, one concern that the authors appropriately acknowledge is that the time data were not necessarily accurate. In most circumstances, the time of application information had to be retrieved by retrospective interview of personnel and involved use of best guess data. Although this record-keeping compliance is understandable under the circumstances, it may point out the need for smarter devices that can automatically record the inflation times and pressures of the tourniquet device. Such considerations have been the focus of a concurrent conference on intelligent medical systems held in conjunction with this assembly. Not only would we have better data collection, but future clinical care devices may be have safer and improved utilization as a result.

In closing, I want to emphasize that the authors have helped to confirm the overall relative safety of appropriate tourniquet use and the lifesaving effect when the device is effectively applied. I personally want to express my admiration for their Herculean pioneer efforts. I think it will significantly impact the clinical care and outcome, not only of our soldiers, but also our families and friends who may someday face the terrible sequelae of trauma in the homeland.
Dr. John F. Kragh (US Army Institute of Surgical Research, Fort Sam Houston, TX): Thanks for the fete, Dr. Pepe. The first aid research regarding tourniquets has been hard work for the team, and it has been productive. We submitted work for review that was lengthy and emphasized morbidity. We aim mortality work for a different venue, and the practical issues for the ATACCC conference where the work was presented are foremost because the users there have already seen the lifesaving capacity of the devices. The practical issues addressed in the work are numerous, and the data fill specific knowledge gaps. The follow-on projects have seen progressive improvements made over the years at the study site, which indicate the overall performance improvement of the whole health care system funneling into the Baghdad hospital is occurring. The positive changes in use indicate that the continued care, education, and refinement of doctrine on the ground in real time are fruitful. The other nations with patients in the system also have seen the results and have taken action to supply more soldiers.

Specifically, the care regarding tourniquets needs to improve regarding recording basic tourniquet information, and this is improving slowly in part because of the current work and the larger overall program. Much work remains to be done regarding education, doctrine refinement, and publication of results. The tourniquet time of duration of use is a good example of what needs attention regarding recording of both on and off times. The duration of use is useful for the surgeon to estimate warm ischemia time, which is associated with complications. However, the overall results of the study show that for the most part, the complications are infrequent and the lifesaving effect is common. The table on tourniquet duration versus complication rate shows that the two are related, which confirms much prior work. We teased out a number of issues for the readers to stew on regarding similar issues so that different readers such as medics and orthopedic surgeon can use the work to base their knowledge on.

Dr. Pepe, you have summarized well the application of the current work in established knowledge, and we thank you for your perspective. It takes such broad perspective to see that this is not just a trauma surgeon issue, this is not just orthopedic or vascular surgery, and this is not just ED nursing or medical care prehospital, but this is first aid. This work touches on all that and lay person training, too, but how it will do so is difficult to predict. The findings need further confirmation before generalizing beyond the military situation. Paramilitary and police situations are likely the most generalizable to the war situation, and civilian care has some applicability, but how much is unclear. In San Antonio, where our home is, prehospital paramedics have used emergency tourniquets recently for civilian trauma. I am unsure of who is teaching them what, but we have seen lives saved here by their use in civilian trauma. More work needs to be done to refine how and when to use the tourniquets in such settings, and history tells us that tourniquet popularity varies.

We thank Dr. Pepe for helping place the work in context so that readers may understand the vital tool that tourniquets can be when used for the right patient at the right time in the right way. 